\title{
Active Flow Control At Low Reynolds Numbers By Periodic Airfoil Morphing
}

\author{
Gareth Jones $^{1 \dagger}$ and Matthew Santer ${ }^{2}$ and George Papadakis ${ }^{3}$ \\ Department of Aeronautics, Imperial College London, London, SW72AZ, United Kingdom \\ Marco Debiasi ${ }^{4}$ \\ Temasek Laboratories, National University of Singapore, Singapore, 117411 \\ ${ }^{\dagger}$ Corresponding author, E-mail: gj11@imperial.ac.uk
}

\begin{abstract}
AIAA Aerospace Sciences Meeting, 4-8 January 2016
This paper investigates the application of a periodically deforming airfoil surface for the purpose of flow control at low Reynolds numbers. A physical model has been fabricated by bonding Macro Fiber Composite (MFC) actuators to the underside of a NACA 4415's suction surface. The results presented build on work by Jones et al. ${ }^{1}$ First, the behavior of the surface when actuated at a range frequencies is investigated through a combination of photogrammetric and laser sensor measurements. Second, the aerodynamic performance of this novel flow control technique is presented. It is shown that when the actuation frequency 'locks-in' to the surface motion significant improvements in performance are observed in a flight regime notorious for poor airfoil behavior.
\end{abstract}

\section{Nomenclature}

$\begin{array}{ll}x & \text { Streamwise coordinate of the wind tunnel } \\ y & \text { Spanwise coordinate of the wind tunnel } \\ z & \text { Vertical coordinate of the wind tunnel } \\ \xi & \text { Axial (chordwise) coordinate of the airfoil chord } \\ \zeta & \text { Normal coordinate of the airfoil chord } \\ l & \text { Local coordinate of the airfoil surface } \\ n & \text { Local normal coordinate of the airfoil surface } \\ c & \text { Model chord } \\ s & \text { Model span } \\ \alpha & \text { Angle of attack } \\ U & \text { Flow velocity } \\ R e & \text { Reynolds number } \\ C_{D} & \text { Drag coefficient } \\ C_{L} & \text { Lift coefficient } \\ V_{A} & \text { Amplitude of sinusoidal actuation } \\ V_{f} & \text { Frequency of sinusoidal actuation } \\ V_{f} & \text { Non-dimensional actuation frequency } \\ \infty & \text { Freestream conditions }\end{array}$

\footnotetext{
${ }^{1}$ Postgraduate researcher, Department of Aeronautics, Imperial College London and Department of Mechanical Engineering, National University of Singapore, Singapore

${ }^{2}$ Senior Lecturer in Structres, Department of Aeronautics, Imperial College London, Senior Member AIAA.

${ }^{3}$ Reader in Aerodynamics, Department of Aeronautics, Imperial College London.

${ }^{4}$ Senior Research Scientist, Temasek Laboratories, National University of Singapore, Singapore, Member AIAA.
} 


\section{Introduction}

B отн military and civilian interest in Unmanned Aerial Vehicles (UAVs) and Micro Air Vehicles (MAVs) Bhas increased significantly in recent years. The availability of very small sensors, video cameras, listening devices and control hardware systems has brought about the prospect of increasingly small aircraft capable of complex missions ranging from surveillance and communication relay to detection of biological, chemical, or nuclear materials. ${ }^{2}$ The combination of small length scales and low velocities results in an operating flight regime of low chord-based Reynolds numbers, $R e_{c}$, of 15,000 to 500,000.

Viscous effects close to the airfoil's surface slow a fluid element down thereby reducing its kinetic energy. Unlike turbulent boundary layers, which can compensate for this by mixing the low momentum fluid with higher momentum fluid at the edge of the boundary layer, the energy of the near-wall flow in a laminar boundary layer remains very low. At sufficiently low Reynolds numbers, such as those experienced by small UAVs and MAVs, laminar boundary layers will form on an airfoil's upper surface and persist beyond the suction peak and into the pressure recovery region - at which point they will encounter an adverse pressure gradient. The very low energy in the near-wall flow leaves such boundary layers incapable of overcoming even modest adverse pressure gradients and as such they are prone to separation even at low angles of attack resulting in high drag and loss of lift - significantly limiting an airfoil's performance. Several years ago, it was recognized that flow control could counter such unfavorable conditions and potentially lead to considerable performance improvements. ${ }^{3}$

Although passive control devices, most commonly vortex generators and surface roughness elements, have proved to be quite effective in delaying flow separation they often introduce a drag penalty when the flow does not separate. In contrast, active control approaches can be largely innocuous except when activated and have the potential for delivering variable power. ${ }^{4}$ Furthermore, if coupling of the control input to instabilities that are associated with flow separation can be achieved substantial control authority is possible.

The drawback of active control, with respect to passive, is that an energy input is required. A primary concern when employing such methods is that the amount of energy input is more than offset by the energy saved. This imposes practical constraints on control methods and actuation systems. Steady suction and blowing techniques were among the first to be investigated and proved very effective at enhancing airfoil performance. However, they require heavy, complex pneumatic systems and a relatively large amount of power to achieve significant aerodynamic benefits. By exploiting the belief that Large Coherent Structures (LCSs) are responsible for transporting momentum across a shear layer and are controllable using periodic motion Seifert et al. ${ }^{5}$ discovered that by superimposing a periodic motion on top of steady blowing, the efficiency of such control methods could be greatly increased.

More recently, the development of new actuation devices and material systems has enabled novel approaches to flow control to be explored and the concept of periodic motion has been further investigated with methods including pulsed vortex generators, ${ }^{3}$ synthetic jets ${ }^{4}$ and oscillating surfaces. ${ }^{6}$ Munday et al. ${ }^{6}$ used a thin, flexible piezoelectric THUNDER actuator, developed at NASA, to morph the surface of an airfoil. When embedded in a surface or attached to flexible structures such actuators provide a distributed force with little power consumption. They are also very light and easy to integrate to the surface of an airfoil thus maximizing their possible aerodynamic gains. Munday et al. performed both static and dynamic morphing tests at angles of attack from $0^{\circ}$ to $9^{\circ}$ at $R e_{c}$ of $2.5 \times 10^{4}$ and $5 \times 10^{4}$. While their static tests did not prove very successful, dynamic actuation was found to significantly reduce flow separation.

The macro fiber composite (MFC) actuator, also developed at NASA, is a more advanced piezoelectric actuator, similar to THUNDER. It consists of rectangular piezoceramic rods sandwiched between layers of adhesive and electrode polyimide film which contains interdigitated electrodes that transfer the applied voltage directly to the rods. ${ }^{7}$ The MFCs can provide distributed dynamic deflection at high frequencies for very little power consumption.

The scope of this paper is to explore the use of MFCs for dynamic actuation of an airfoil surface. Their ability to perform the dynamic motion will be presented along with their effects on the aerodynamic performance of a specially fabricated NACA 4415 airfoil. This work builds on work previously presented by Jones et al. ${ }^{1}$ and includes more detailed analysis of the surface motion and flow behavior. 


\section{Experimental Setup}

\section{II.A. Fabrication}

An airfoil model based on a NACA 4415 has been fabricated for investigating the effect of dynamic surface morphing by MFC actuators. The leading edge, trailing edge and pressure surface were machined from PVC to true NACA 4415 coordinates with a tolerance of $\pm 0.1 \mathrm{~mm}$, while the suction surface was fabricated separately to accommodate the desired deformations. To achieve a deformable surface, two MFC actuator patches (Smart Material M-8557-P1) were bonded to a $0.25 \mathrm{~mm}$ thick sheet of Titanium with a slow drying epoxy resin. A vacuum bag was placed over the skin while the epoxy set to remove any pockets of air between the Titanium and MFCs, ensuring a strong, clean bond. The resulting skin can be seen in figure 1a. The fully formed model has a chord, $c$, of $150 \mathrm{~mm}$ and span, $s$, of $158 \mathrm{~mm}$ and can be seen in figure $1 \mathrm{~b}$.

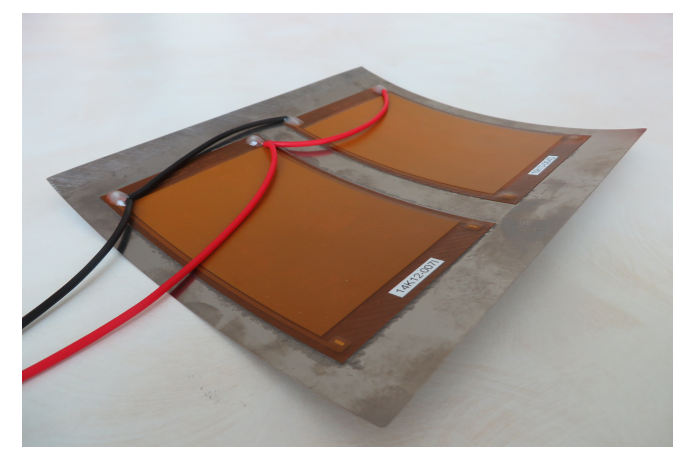

(a)

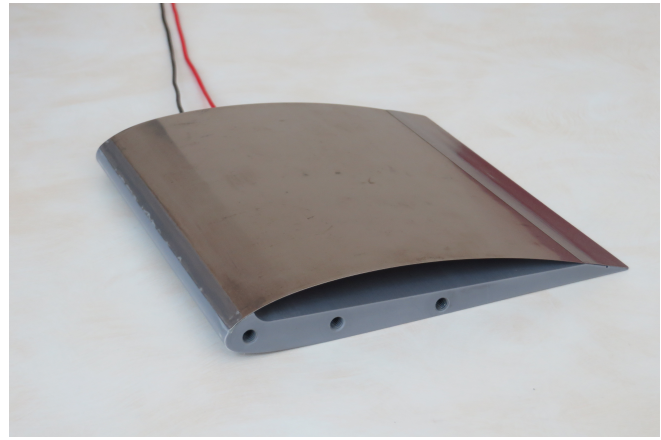

(b)

Figure 1: Airfoil model for testing: a) inner side of a titanium skin with MFC patches; b) assembled model.

The skin was rigidly attached to the body of the aerofoil at the leading edge. At the trailing edge a thin slide joint was used to allow for small displacements in the longitudinal direction that occurred when the surface was deformed. ${ }^{8}$ In slight variation to previous models presented by Debiasi et al. ${ }^{7-9}$ which were mainly meant for static deformations, the slide on this model was located closer to the trailing edge offering a larger deformable area and therefore increasing the dynamic range.

\section{II.B. Experimental Apparatus}

The aerodynamic characteristics of the model have been tested in the closed-loop, subsonic wind tunnel at the NUS Temasek Laboratories, figure 2a, which has a $600 \mathrm{~mm}$ square test section with a length of $2 \mathrm{~m}$. The test section is connected to the exit of the wind-tunnel nozzle which has a 12:1 contraction ratio. The turbulence intensity level of the wind-tunnel freestream is less than $0.25 \%$ at velocities of less than $15 \mathrm{~m} / \mathrm{s}-$ which is the range of interest in this paper. Since the span of the model is smaller than the width of the test section, the model was mounted vertically on a turntable in the test section floor and a splitter plate was installed $160 \mathrm{~mm}$ above it. The turntable allows precise positioning (within $\pm 0.05^{\circ}$ ) of the angle of attack, $\alpha$, of the model. A boundary-layer ingestion slot with a sharp leading edge spanning $72 \%$ of the test-section width was utilized to maintain a floor boundary layer roughly as thin as the one on the surface of the splitter plate. The leading edge of the model was positioned $400 \mathrm{~mm}$ downstream of the leading edge of the ingestion slot, at which location the boundary-layer thickness of the empty test section with $U_{\infty}=10 \mathrm{~m} / \mathrm{s}$ has been measured to be less than $3 \mathrm{~mm}$. The mounting can be seen in figure $2 \mathrm{~b}$. 

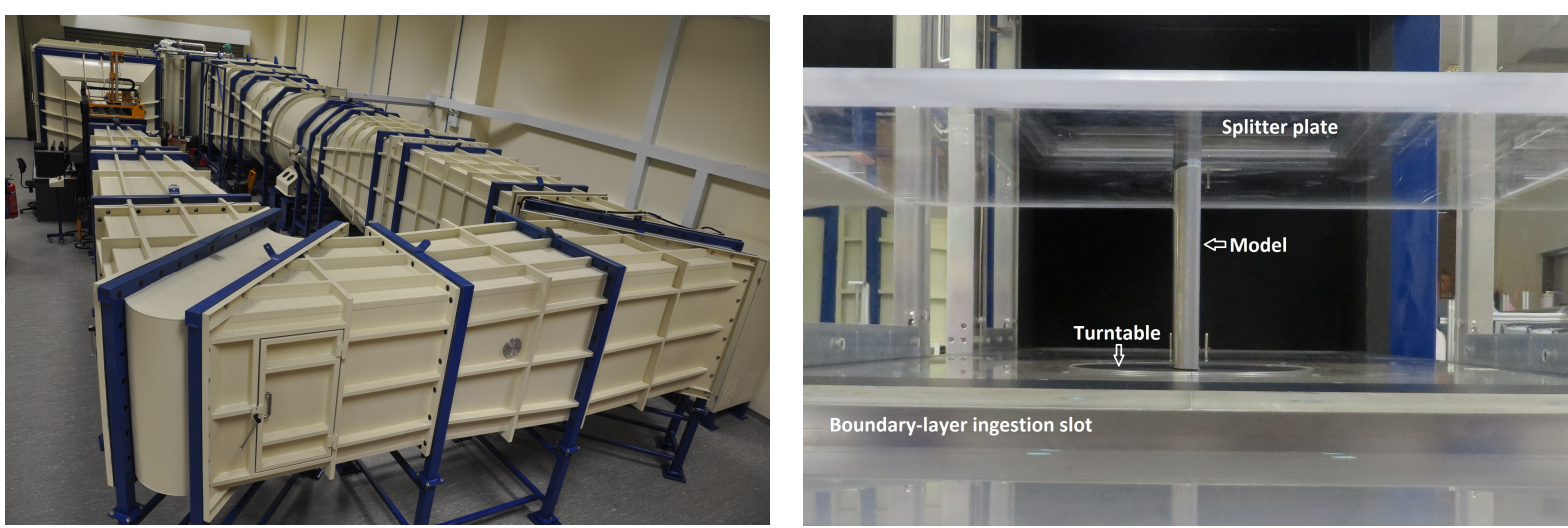

Figure 2: Experimental facilities. a) closed-loop, subsonic wind tunnel; b) model mounting.

The turntable on which the model was mounted incorporated a force balance consisting of a Gamma ATI SI-32-2.5 piezoelectric gauge capable of measuring the forces and moments along three perpendicular axes. The two axes aligned with the axial (chordwise) and the normal coordinates of the airfoil were used to measure the axial and the normal forces generated by the model. The third axis, coinciding with the axis of rotation of the turntable and aligned in the vertical direction of the tunnel, passed through the airfoil mid-chord point (c/2) and was used to measure the pitching moment. The balance was factory calibrated and the corresponding conversion factors are stored in the acquisition unit used with it so the values of the forces and moments obtained are already corrected. The range (and resolution) of the measured forces and moment are $32\left( \pm 6 \times 10^{-3}\right) \mathrm{N}$ and $2.5\left( \pm 5 \times 10^{-4}\right) \mathrm{Nm}$, respectively. For each measurement, 10 seconds worth of samples were acquired at a minimum of 16 samples per actuation cycle $(1.25 \mathrm{kHz}$ without actuation). The lift and the drag coefficients were calculated at each angle of attack from the corresponding values of the chordwise and normal forces according to:

$$
C_{L}=\frac{F_{\eta} \cos (\alpha)-F_{\zeta} \sin (\alpha)}{\frac{1}{2} \rho U_{\infty}^{2} c s}, C_{D}=\frac{F_{\zeta} \cos (\alpha)+F_{\eta} \sin (\alpha)}{\frac{1}{2} \rho U_{\infty}^{2} c s}
$$

The subscripts in equation (1) relate to the axis convention in figure 3. Cartesian coordinates $x, y$ and $z$ refer to the streamwise, cross-stream and spanwise directions respectively with the origin at the aerofoil's leading edge. A rotating coordinate system with an origin at the mid-chord corresponds to the axial $(\zeta)$ and normal $(\eta)$ coordinates of the aerofoil as $\alpha$ changes. Furthermore, a local coordinate system is defined on the aerofoil surface to aid post-processing — $n$ and $l$ being the normal and longitudinal directions respectively.

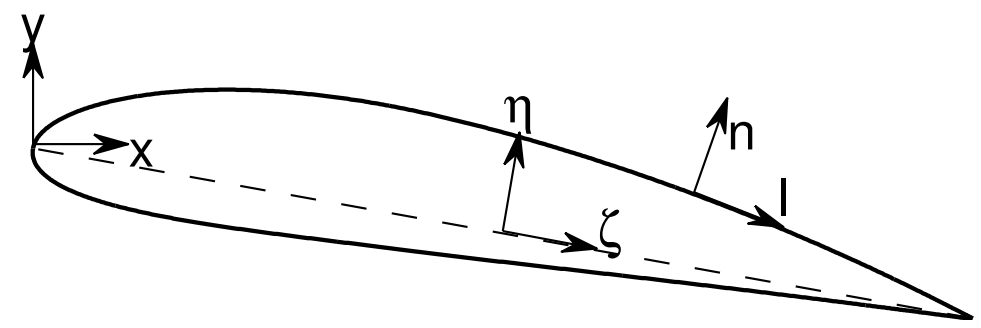

Figure 3: Axis convention and coordinate notation for mounted aerofoil.

Prior to the aerodynamic investigation, a series of tests were carried out to understand the dynamic response on the skin at different voltage amplitudes and frequencies. The deformation of the skin was measured using photogrammetry - a method of obtaining quantitative measurements from photographs. Coded targets were placed on the airfoil surface every $0.1 c$ in both the $l$ and $z$ directions providing 81 points on the morphing section of the surface as can be seen in figure 4. Nine points were also placed on the nondeforming leading and trailing edges to provide a stationary reference. Images were captured at between 1000 and 2000 frames per second at 8 different locations around the airfoil using a Photron Fastcam SA-Z with image resolution of $1024 \times 1024$ pixels. This resolution meant that the center of the coded targets were roughly 10 pixels across which is necessary for the processing software to accurately capture their location. 
A Micro-Epsilon optoNCDT 1710-50 laser displacement sensor was also used to obtain time-resolved point displacements. This unit has a measuring resolution of $50 \mu \mathrm{m}( \pm 5 \mu \mathrm{m})$ and its measuring range allows it to be placed outside the wind-tunnel thus enabling measurements of the skin deformation in the flow. These measurements were acquired at $2.5 \mathrm{kHz}$ and acted as a benchmark for verifying the photogrammetry measurements.

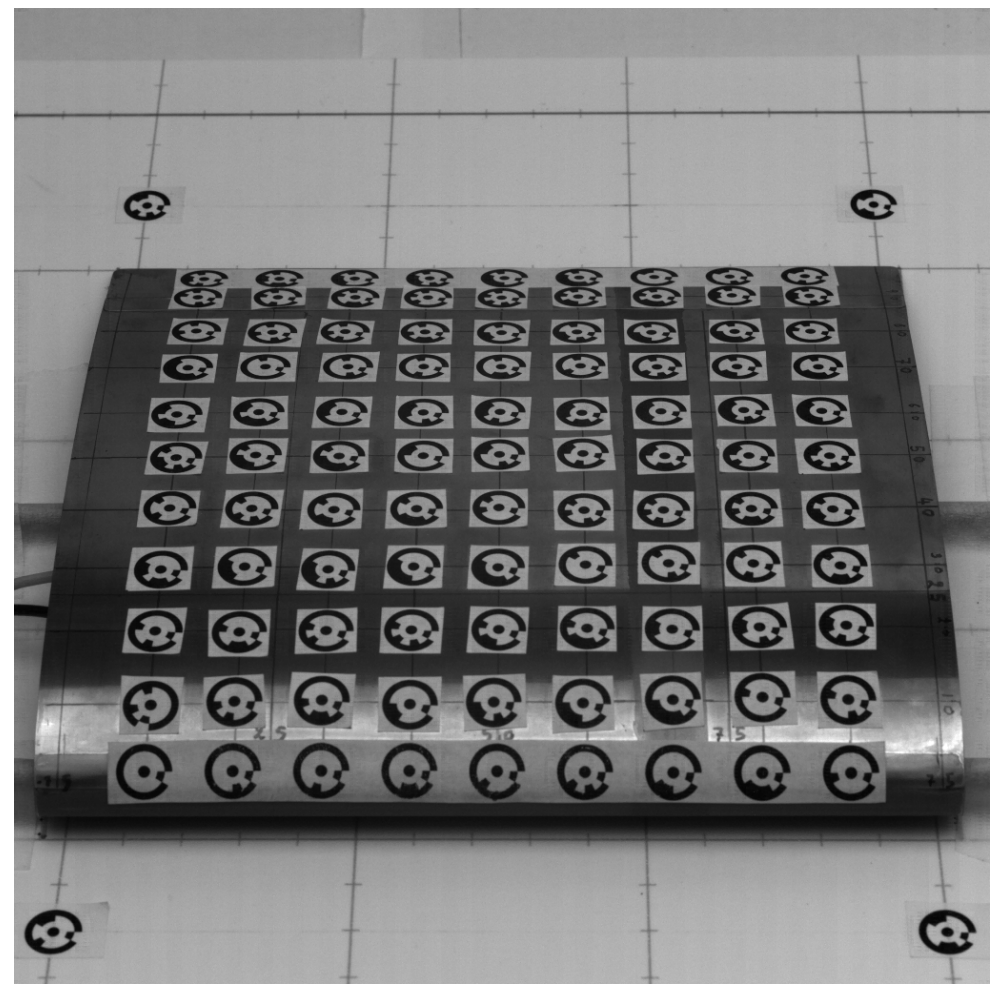

Figure 4: Positioning of coded targets on airfoil surface.

Flow-field velocity measurements were obtained using a two-velocity-component PIV system. The flow was uniformly seeded with water-based particles from a SAFEX fog generator. Droplets were produced in the average size SMD of $1 \mu \mathrm{m}$ whose reflections correspond to no more than 3 pixels in the captured images, allowing a good resolution of the particle displacement when cross-correlation methods are adopted. A dualhead Litron DualPower 200-15 Nd:YAG laser operating at the second harmonic (532 nm) at approximately $150 \mathrm{~mJ}$ per pulse was used in conjunction with sheet-forming optics to form a thin sheet $(\approx 1 \mathrm{~mm})$ on the $x-y$ plane at $70 \%$ along the airfoil span. Two images corresponding to the pulses from the laser were acquired by a $2048 \times 2048$ pixels HiSense 620 camera which viewed the streamwise laser sheet orthogonally over the entire field of view. A computer with dual Intel Core processors was used for data acquisition. The acquired images were divided into $32 \times 32$ pixel interrogation windows which contain at least 3 seeding particles each. For each image, subregions were adaptively cross-correlated using multi-pass processing with a final $50 \%$ overlap that gives a final interrogation area of $16 \times 16$ pixels after processing. Based on the flow velocity and the size of the interrogation area, the time separation between the two laser flashes was varied between 100 and $250 \mu \mathrm{s}$ such that the maximum displacement of a particle in the region of interest is no more than $25 \%$ of the interrogation window between pulses. This is optimum for the PIV processing software to calculate accurately the particle velocity. The resulting vector fields were post-processed to remove remaining spurious vectors. A Zeiss $50 \mathrm{~mm} \mathrm{f} / 2.0$ macro lens which provided a $225 \times 225 \mathrm{~mm}$ field of view corresponding to a velocity vector grid of $127 \times 127$ points with resolution of approximately $110 \mu \mathrm{m}$ per pixel. For each acquisition 300 images were taken for statistical averaging.

Profiles of the wake velocity were also obtained at the mid-span location, $1.25 c, 1.50 c$ and $1.70 c$ downstream of the leading edge using a single, miniature-wire hotwire probe. $2^{17}$ samples at $6 \mathrm{kHz}$ were acquired at 49 different cross-stream locations between $\pm 0.43 c$ in the $y$-direction. The velocity was calibrated with an $1 \%$ error using a dedicated Dantec Dynamics velocity calibrator. 


\section{Preliminary Results}

\section{III.A. Surface Motion}

\section{III.A.1. Static Deformation}

Figure 5 shows a comparison between true NACA 4415 coordinates and the non-actuated airfoil geometry obtained from photogrammetric data. The raw images, such as that seen in figure 4, were processed using 'Photomodeler.' The surface appears slightly flatter than the reference geometry but on the whole the model appears to be representative of a true NACA 4415. Furthermore, the points on the surface calculated by the photogrammetry software are correctly positioned at every $0.1 c(15 \mathrm{~mm})$ with an maximum error of $9 \%$ $( \pm 1.35 \mathrm{~mm})$, well within a reasonable error associated with the coded targets being positioned by hand. This gives confidence that the photogrammetry is correctly capturing the 3D points.

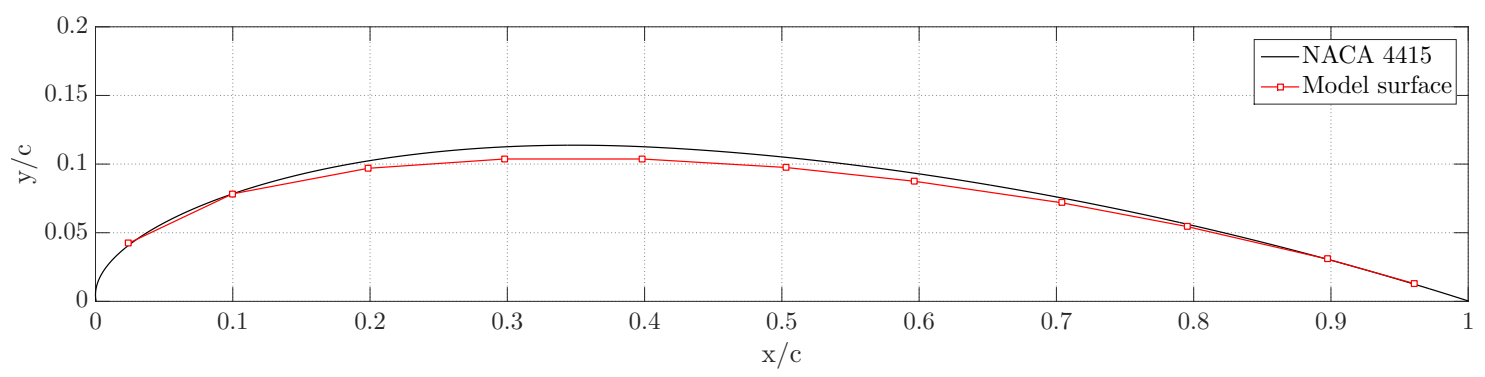

Figure 5: Physical model compared with NACA 4415 coordinates.

A voltage is applied to the MFCs via a Smart Materials HVA 1500/50-2 high-voltage amplifier which has a gain of $200 \mathrm{~V} / \mathrm{V}$ and can accept input signals in the range of $-2.5 \mathrm{~V}$ to $7.5 \mathrm{~V}$. These values are amplified to $-500 \mathrm{~V}$ and $1500 \mathrm{~V}$ respectively and correspond to the operational range of the actuators. Applying a negative voltage to the MFC will cause an outward deformation and a positive voltage will deform the skin inward. The maximum achievable displacement of the skin in the outward direction occurs when the actuators are driven with a voltage of $-500 \mathrm{~V}$ and the maximum inward displacement occurs at $1500 \mathrm{~V}$ as can be seen in figure 6 .

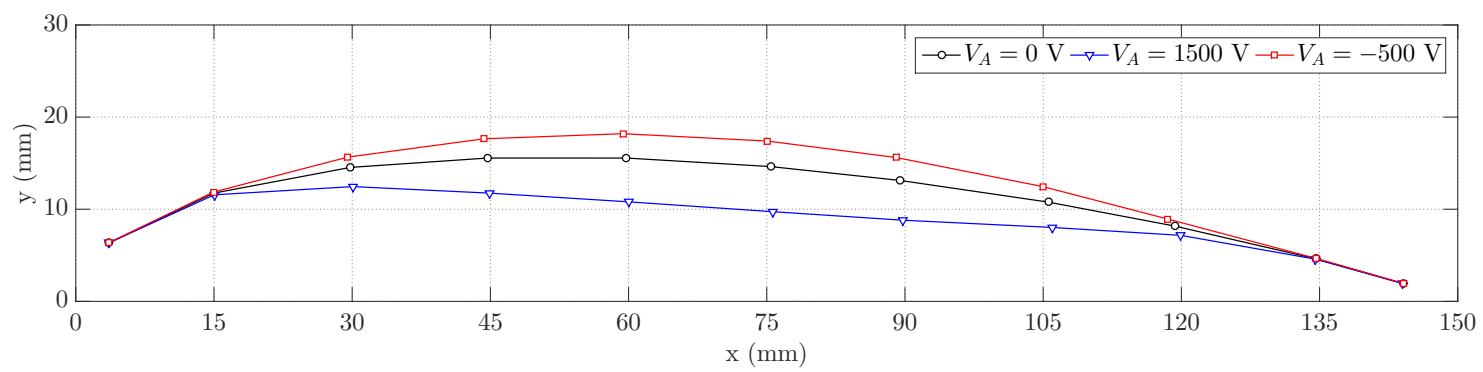

Figure 6: Deformed surfaces compared with unactuated model.

The non-actuated surface is considered as the origin and a displacement in the outward direction is considered positive. Three-dimensional reconstructions of the deformed surface obtained from photogrammetric data, shown in figure 7 , reveal the deformation to be highly 2-dimensional. 

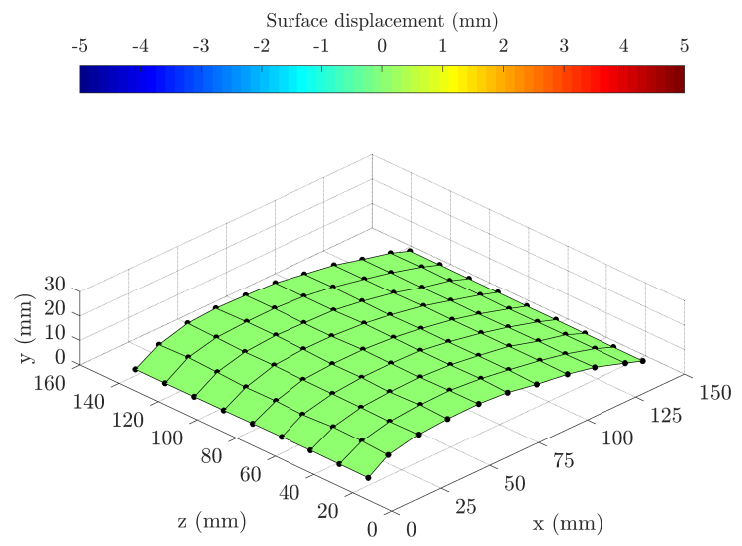

(a)

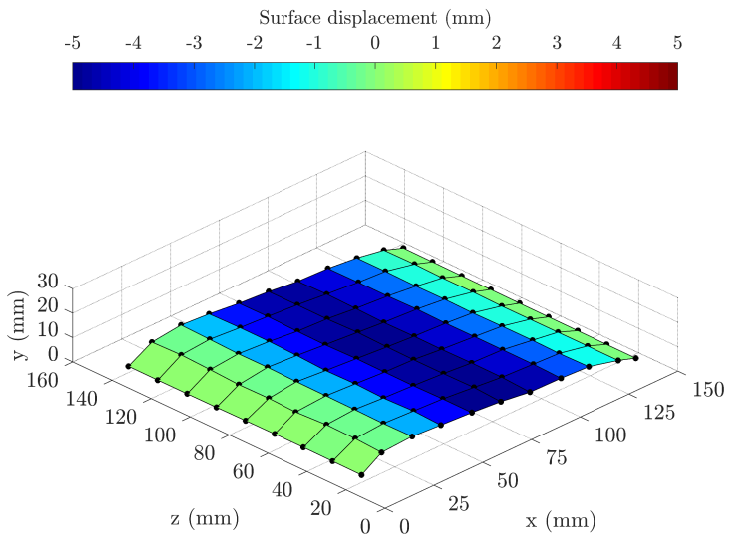

(b)
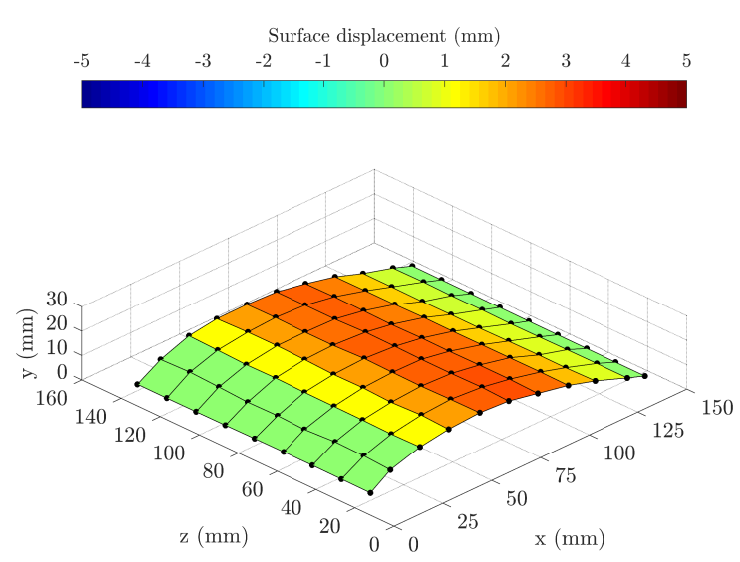

(c)

Figure 7: 3D photogrammetry images of model: a) 0 V DC; b) 1500 V DC; c) -500 V DC. Colored by displacement from unactuated geometry

\section{III.A.2. Dynamic Deformation}

The dynamic behavior of the skin was investigated by driving the MFCs with a series of sinusoidal voltage signals of different amplitudes, $V_{A}$ and frequencies, $V_{f}$, defined as

$$
V(t)=V_{A} \cdot \sin \left(2 \pi V_{f} t\right)
$$

The leading and trailing edge of the morphing section of the surface are known to be located at $0.07 \mathrm{c}$ and $0.93 \mathrm{c}$ respectively and the time-dependent displacement of the skin was recorded at more 4 locations: $0.25 \mathrm{c}, 0.4 \mathrm{c}, 0.5 \mathrm{c}$ and $0.7 \mathrm{c}$. Figure 8 shows the peak-to-peak displacements at the aforementioned locations. It was important to know how a pressure distribution over the surface affected the response of the skin when immersed in a flow both from the point of view of the aerodynamic application but also because it was not possible to perform the photogrammetry in situ. Therefore measurements were taken in both still and moving air at $R e_{c}=5 \times 10^{4}$. 


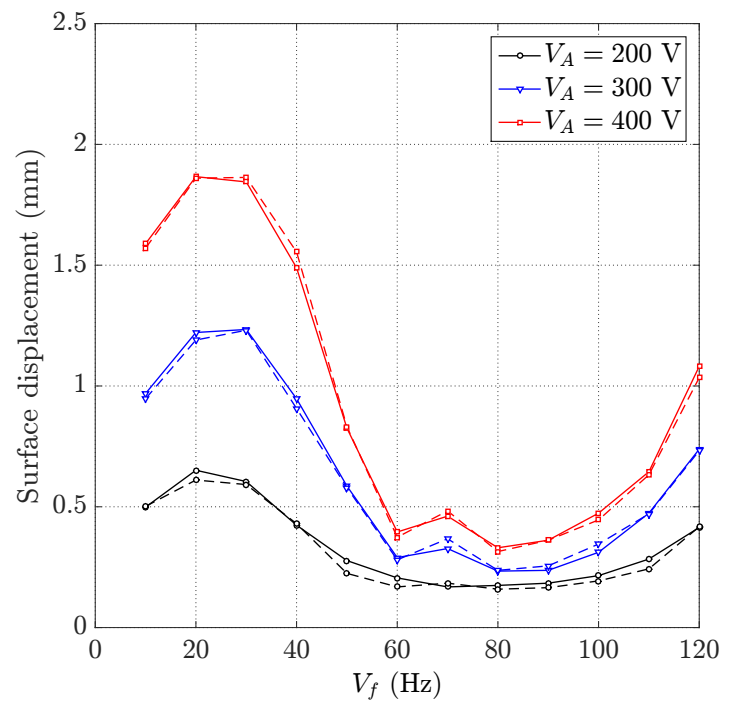

(a)

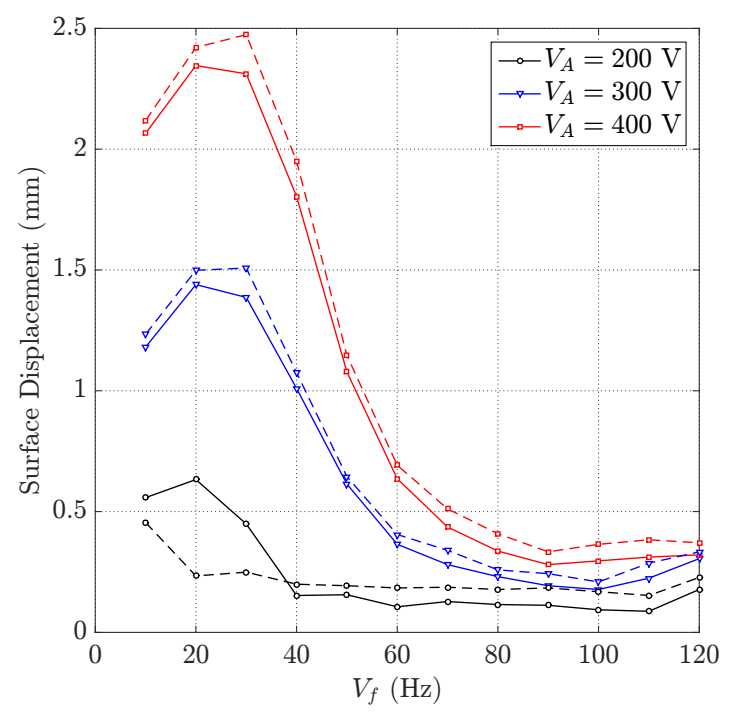

(c)

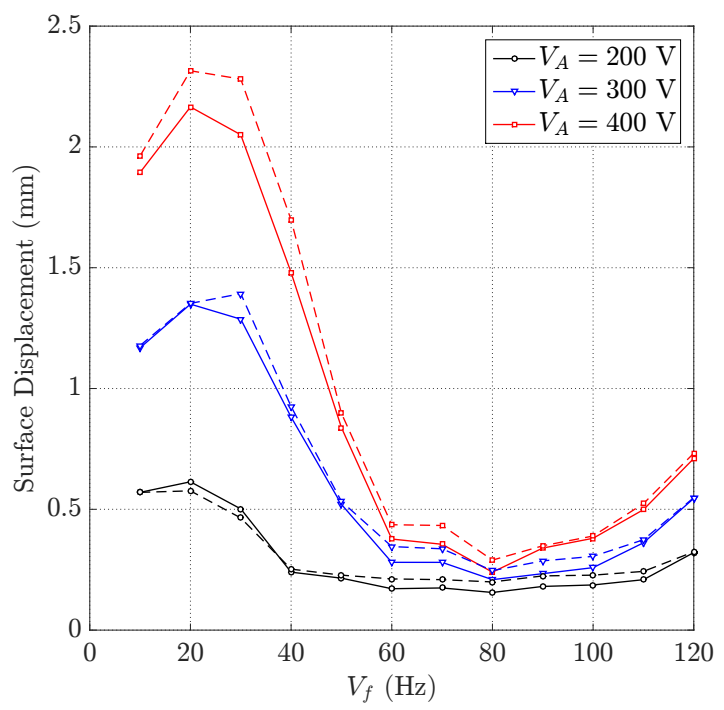

(b)

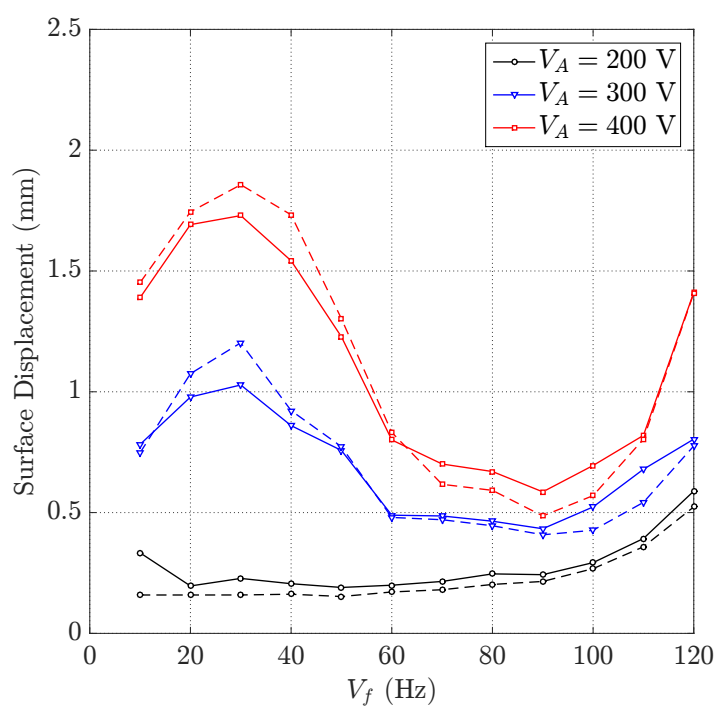

(d)

Figure 8: Peak-to-peak displacement of the surface with $R e_{c}=0$ (Solid) and $R e_{c}=50,000$ (Dashed) at a) $0.25 c$; b) $0.4 c$; c) $0.5 c$; and d) $0.7 c$.

Both amplitude and frequency of the input signal have significant effect on the dynamic behavior of the skin but the aerodynamic forces in moving air appear to have little impact at on the surface motion when $R e_{c}=5 \times 10^{4}$. As a general rule, the higher the amplitude of sinusoidal voltage, the greater the peak-to-peak displacement. At frequencies above $30 \mathrm{~Hz}$ the amplitude of displacement drops significantly at all measured locations. This was expected and had been observed in previous models ${ }^{7}$ and is attributed to resonance occurring between 30 and $40 \mathrm{~Hz}$. However, the amplitude begins increasing again at around $90 \mathrm{~Hz}$. From figure 9 this can be attributed to a change in the vibration mode. 


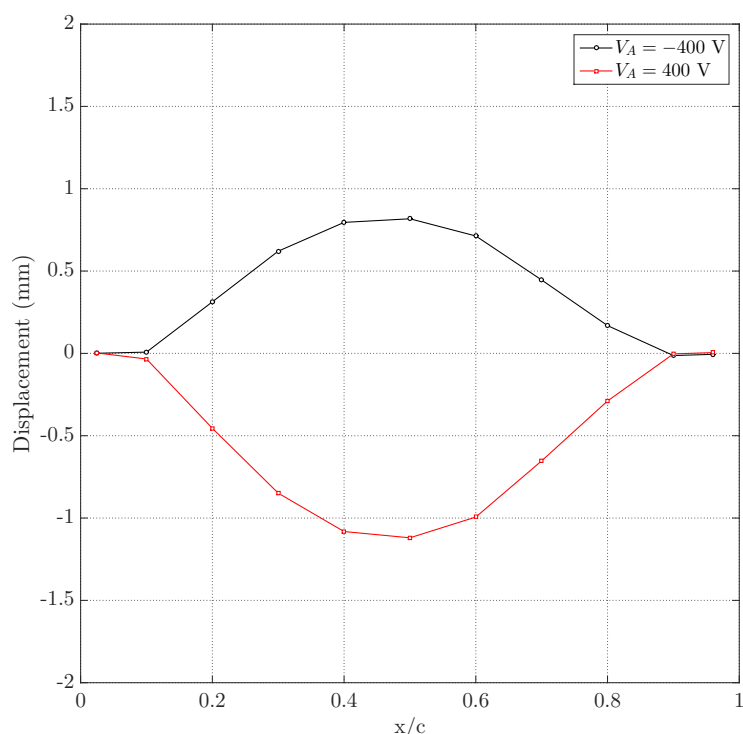

(a)

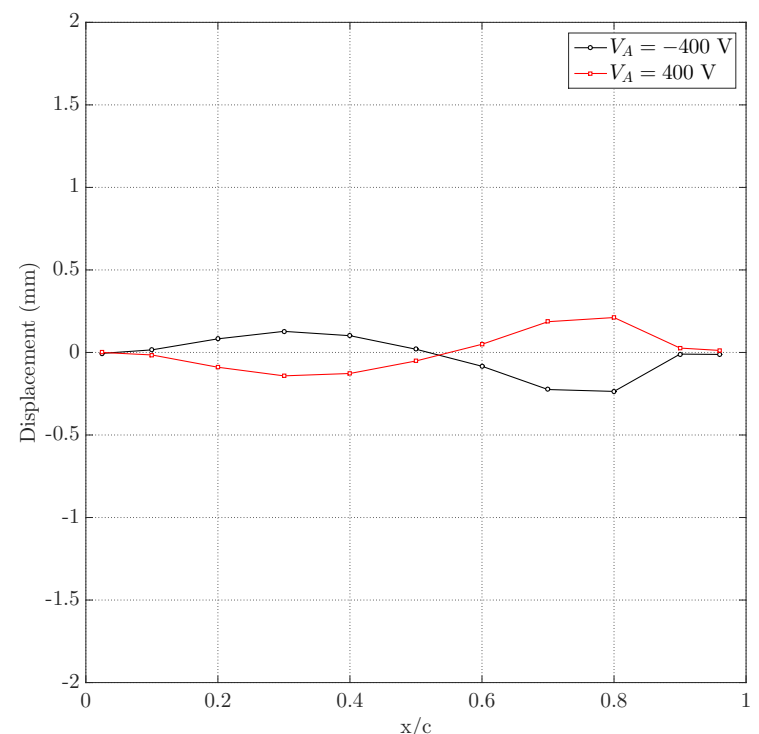

(b)

Figure 9: Peak displacements of the actuated surface with reference to the unactuated geometry: a) $V_{f}=10$ $\mathrm{Hz}$ b) $V_{f}=100 \mathrm{~Hz}$.

To check how accurately the photogrammetry captured the dynamic surface motion, a time trace of 10 cycles at $V_{f}=100 \mathrm{~Hz}$ - considered the most challenging to capture owing to small displacements and complicated vibration mode shape - is compared with the laser displacement data in still air at two different midspan locations in figure 10. As the laser measurements in still air and moving air are very similar, it was considered acceptable to perform the photogrammetry experiments outside the tunnel - which was necessary for optical access to the morphing surface.

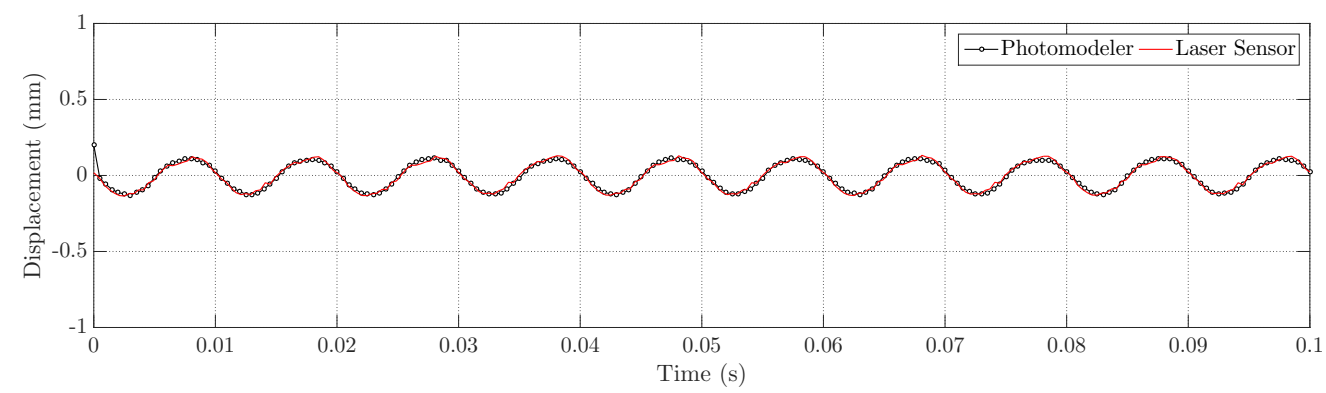

(a)

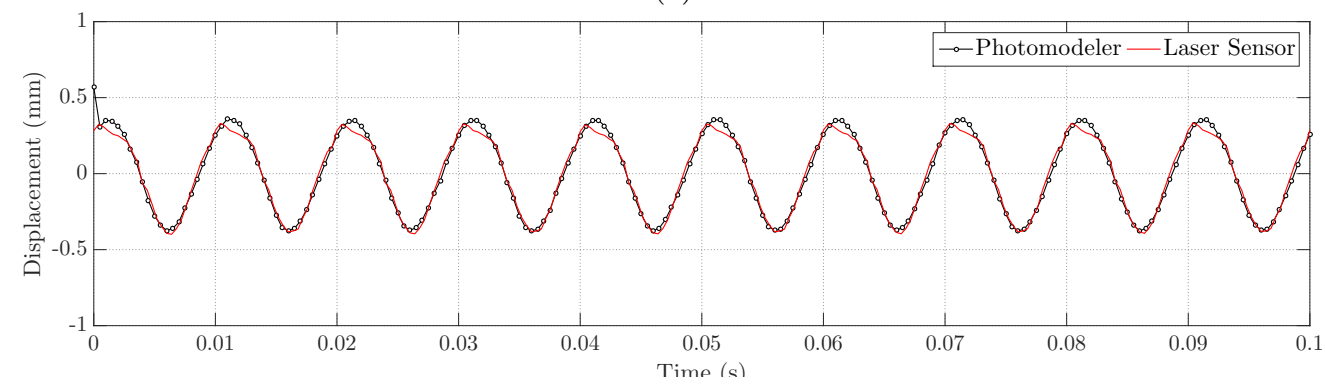

(b)

Figure 10: Comparison of photogrammetry data and laser displacement sensor at midspan when $V_{f}=100$ Hz: a) $0.4 c \mathrm{~Hz}$; b) $0.7 c$.

The photogrammetry is in very good agreement with the laser displacement sensor and can therefore be considered capable of capturing the dynamic behavior of the surface. 
In this paper, it is the effect of frequency that is to be investigated. However, from figure 8 it is clear that this cannot be achieved by simply varying $V_{f}$ because this causes large changes in the peak-to-peak displacement of the surface. Therefore $V_{A}$ was also modified with $V_{f}$, according to figure 8 , to keep the peak-to-peak surface displacement at roughly $0.5 \mathrm{~mm}$ at a location of $0.4 c$ along the chord, thus isolating the effect of frequency.

\section{III.B. Aerodynamic Measurements}

The effect of actuation frequency on aerodynamic performance was investigated by testing three different values of $V_{f}: 10 \mathrm{~Hz}, 40 \mathrm{~Hz}$ and $70 \mathrm{~Hz}$, which can be non-dimensionalized with $c$ and $U_{\infty}$ as:

$$
V_{f^{+}}=\frac{V_{f} c}{U_{\infty}}
$$

This provided a set of reduced frequencies, $V_{f^{+}}$, of $0.3,1.1$ and 2.0 respectively.

\section{III.B.1. Time-Averaged Results}

Time-averaged flow fields obtained from PIV data are displayed in figures 11-14. Baseline data at $\alpha=0^{\circ}, 5^{\circ}$, $10^{\circ}$ and $15^{\circ}$, taken when the airfoil surface was stationary, shows that boundary layer separation is present at all angles of attack. When $\alpha=0^{\circ}$ this occurs at approximately $0.6 c$ and moves upstream to $0.5 c, 0.4 c$ and $0.2 c$ when $\alpha$ is increased to $5^{\circ}, 10^{\circ}$ and $15^{\circ}$ respectively. This agrees with previous studies of a laminar separation. ${ }^{10-12}$ It is also a good demonstration of the inability of a laminar boundary layer to overcome even modest adverse pressure gradients as separation occurs without reattachment even when $\alpha=0^{\circ}$. A much larger region of separation is present when $\alpha=15^{\circ}$ where the airfoil appears to be stalled.

At the lowest tested frequency, $V_{f^{+}}=0.3$, the flow fields at all angles appear very similar to the baseline case, suggesting that actuation at this reduced frequency is incapable of triggering any changes to the flow development. However, when $V_{f^{+}}=2.0$, the separation regions at all angles are significantly smaller, most noticeably when $\alpha=15^{\circ}$. The intermediate value of $V_{f^{+}}=1.1$ seems more complicated though. The separation regions at $0^{\circ}$ and $5^{\circ}$ show only a minor reduction in separation when compared to the baseline case, however at $10^{\circ}$ and $15^{\circ}$ this reduction is far more significant thus suggesting that this particular frequency has a greater effect at higher angles of attack. 


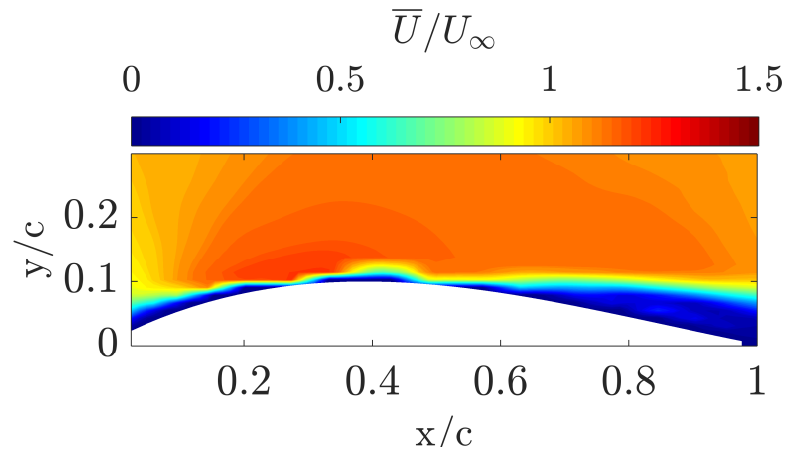

(a)

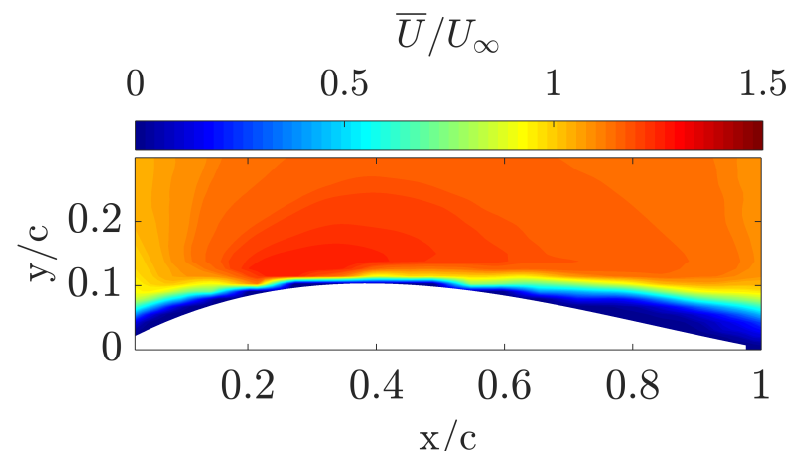

(c)

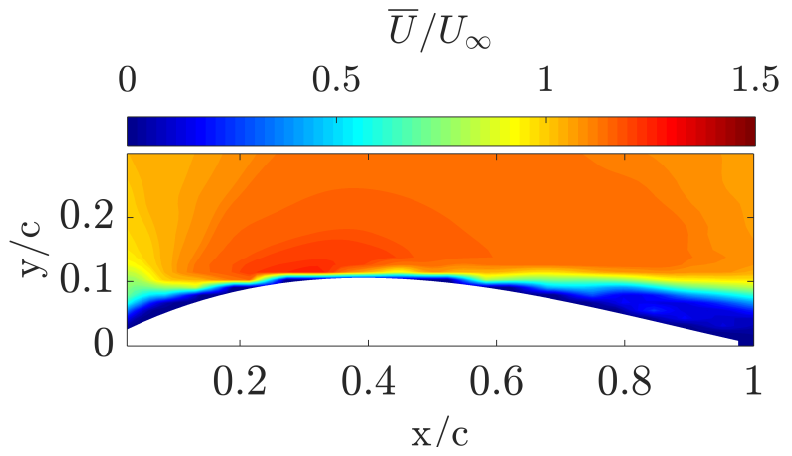

(b)

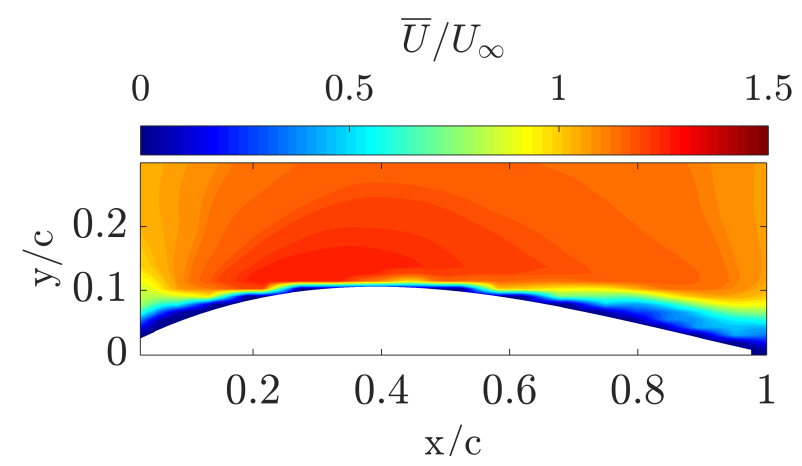

(d)

Figure 11: Flow fields from PIV measurements at $\alpha=0^{\circ}$ : a) $V_{f^{+}}=0$; b) $V_{f^{+}}=0.3$; c) $V_{f^{+}}=1.1$; d) $V_{f^{+}}=2.0$. 


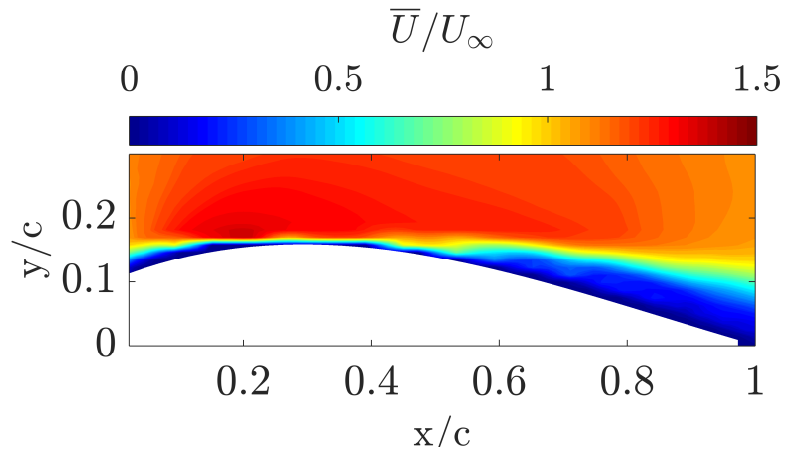

(a)

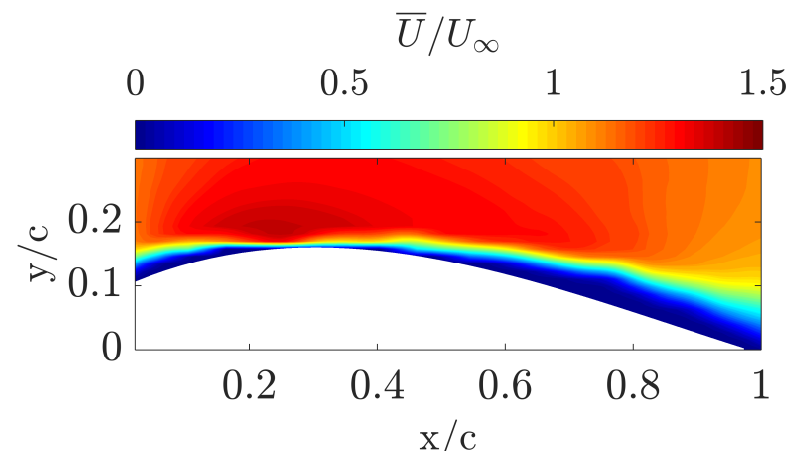

(c)

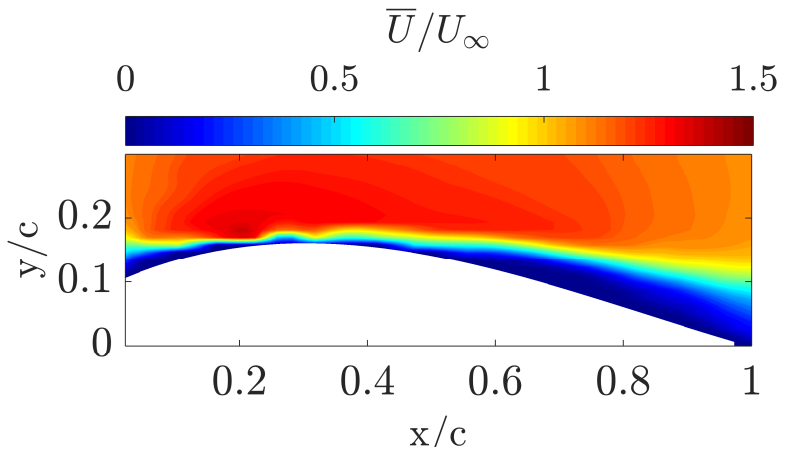

(b)

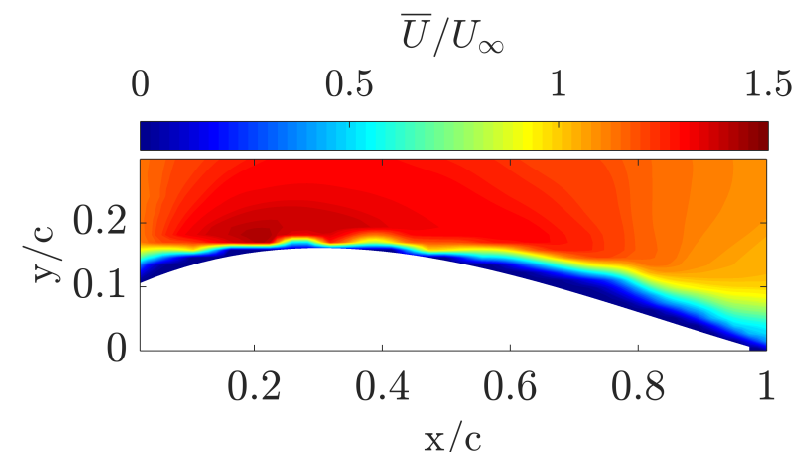

(d)

Figure 12: Flow fields from PIV measurements at $\alpha=5^{\circ}$ : a) $V_{f^{+}}=0$; b) $V_{f^{+}}=0.3$; c) $V_{f^{+}}=1.1$; d) $V_{f^{+}}=2.0$. 

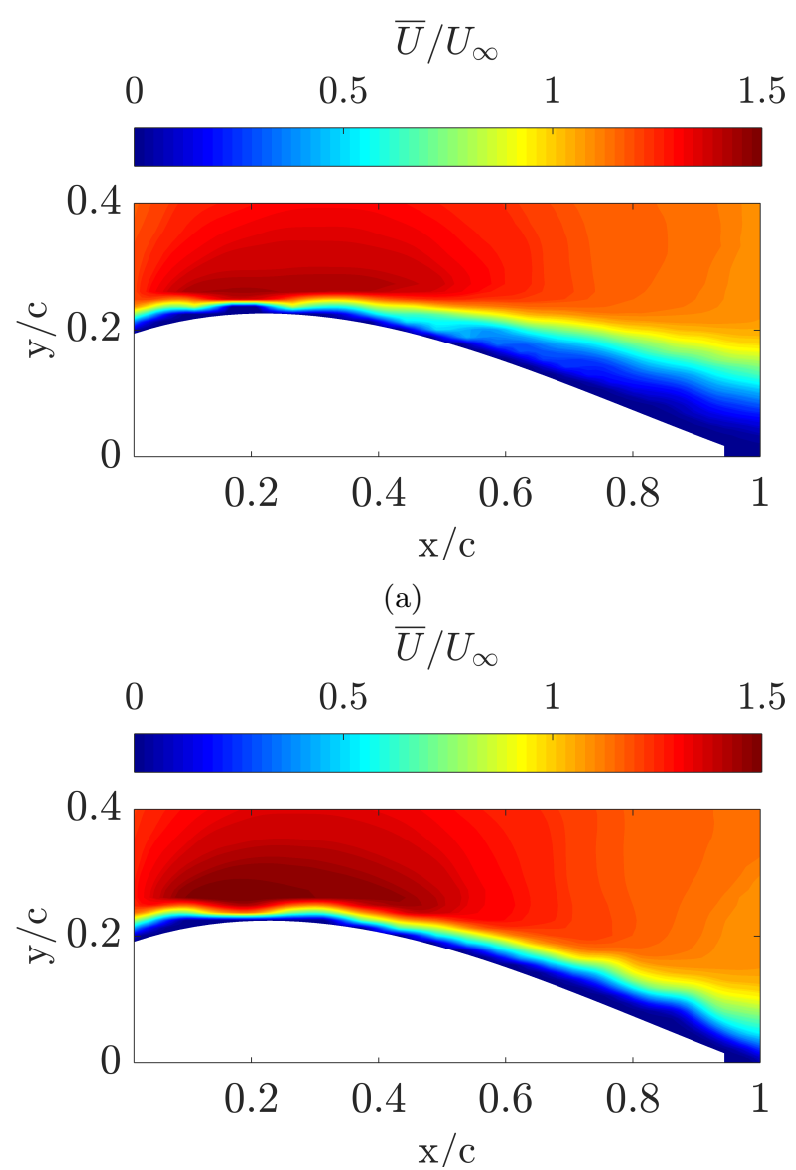

(c)

1.5

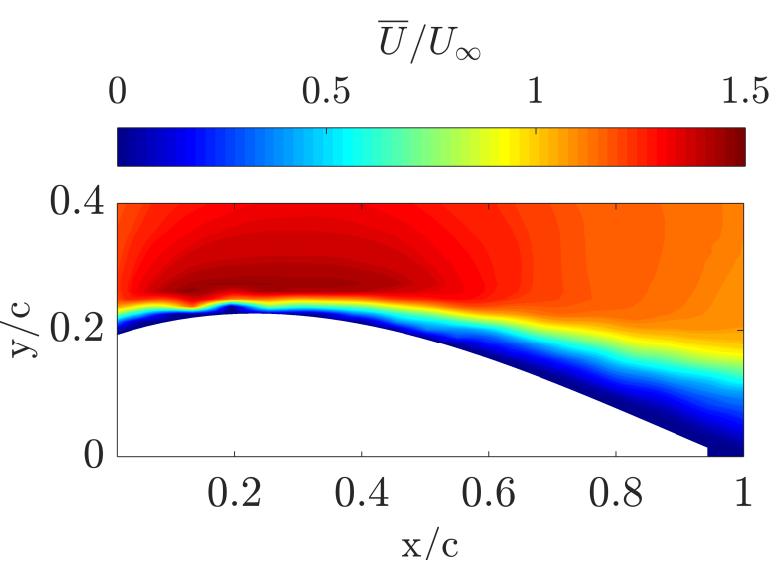

(b) $\bar{U} / U_{\infty}$
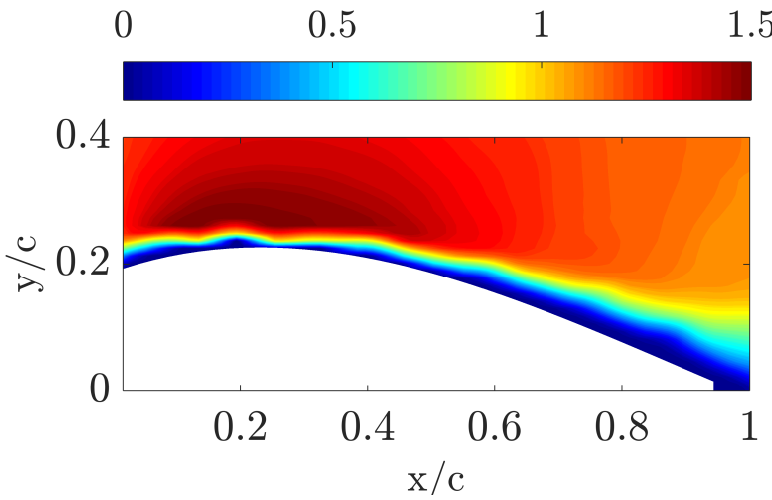

(d)

Figure 13: Flow fields from PIV measurements at $\alpha=10^{\circ}$ : a) $V_{f^{+}}=0$; b) $V_{f^{+}}=0.3$; c) $V_{f^{+}}=1.1$; d) $V_{f^{+}}=2.0$. 

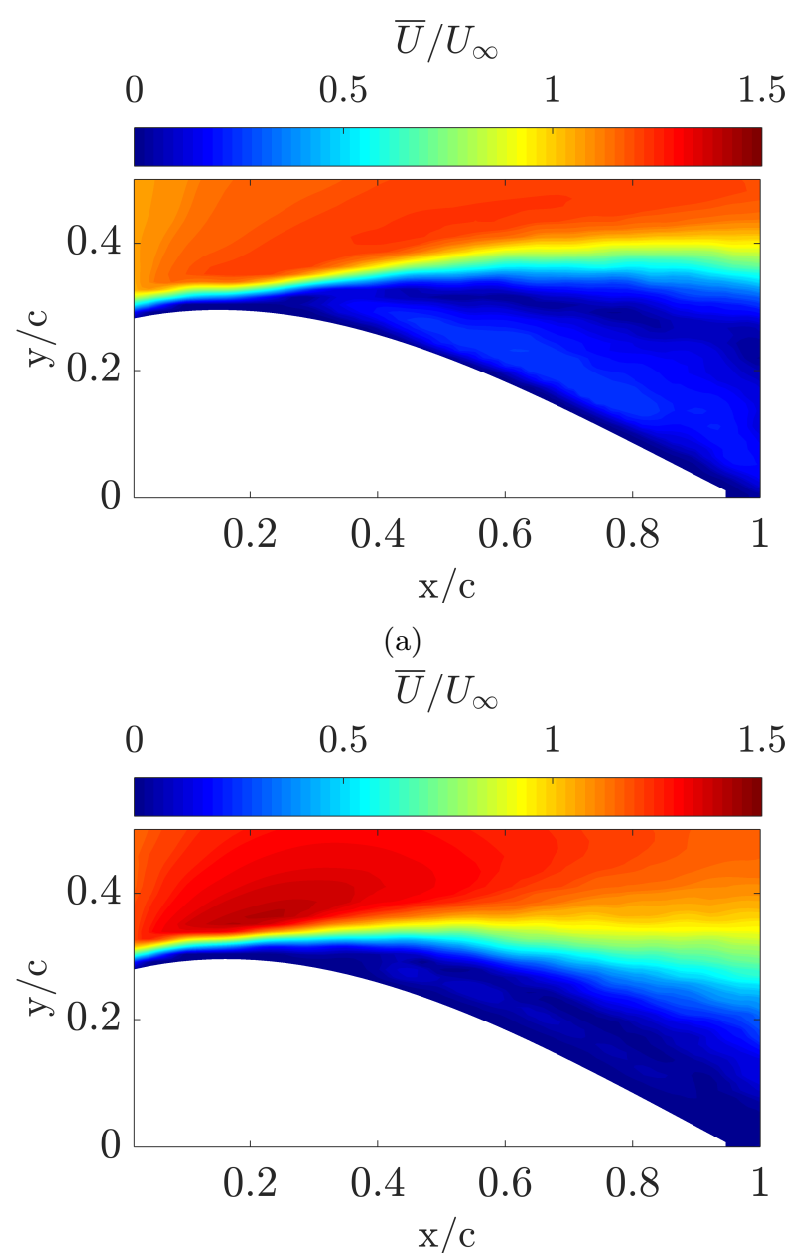

(c)

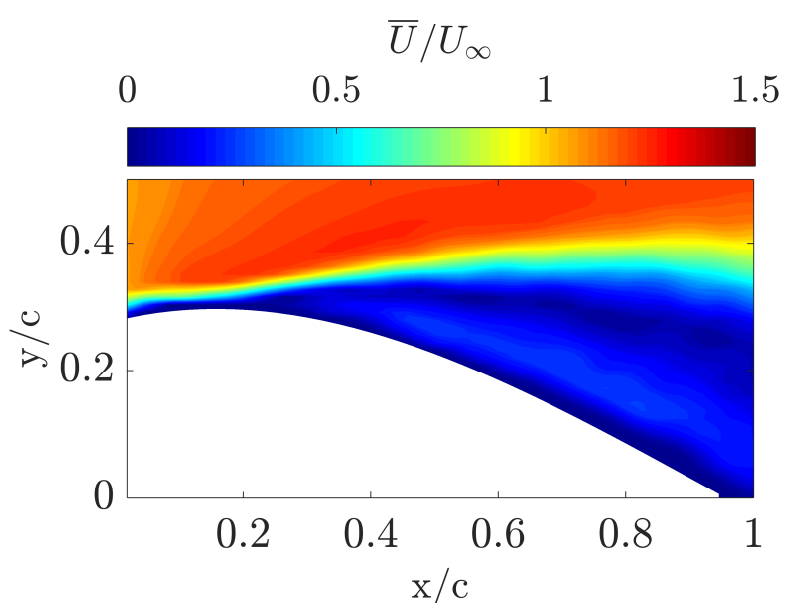

(b)

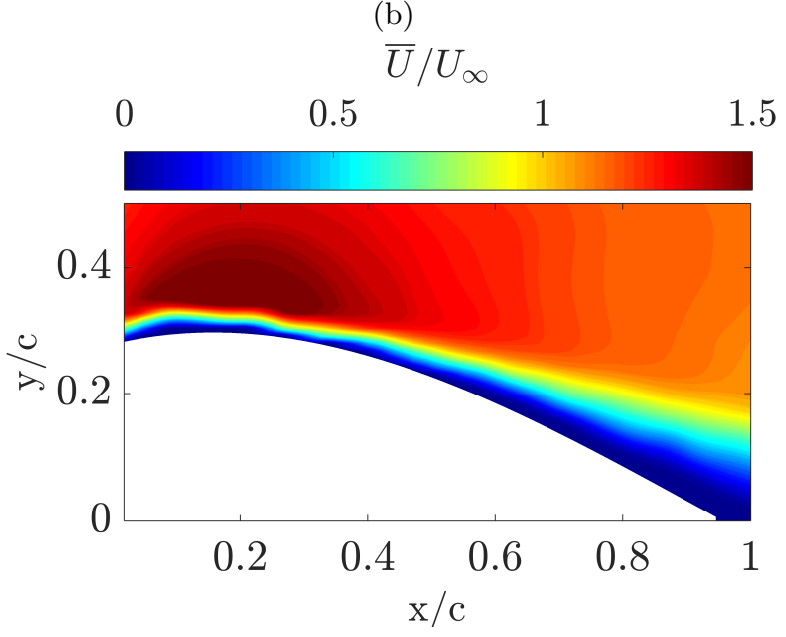

(d)

Figure 14: Flow fields from PIV measurements at $\alpha=15^{\circ}$ : a) $V_{f^{+}}=0$; b) $V_{f^{+}}=0.3$; c) $V_{f^{+}}=1.1$; d) $V_{f^{+}}=2.0$.

As well as the flow around the airfoil, the wake was also investigated at several streamwise locations and the time-averaged velocity profiles when $\alpha=0^{\circ}$ can be seen in figure 15. A very clear reduction in the magnitude of the velocity deficit is observed at actuation frequencies of $V_{f^{+}}>1$ but when $V_{f^{+}}=0$ and 0.3 the profiles are almost identical. When $V_{f^{+}}=1.1$ the reduction in velocity deficit is modest however when $V_{f^{+}}=2.0$ it is much more significant. This is in agreement with what has been described from the flow field measurement around the airfoil. 


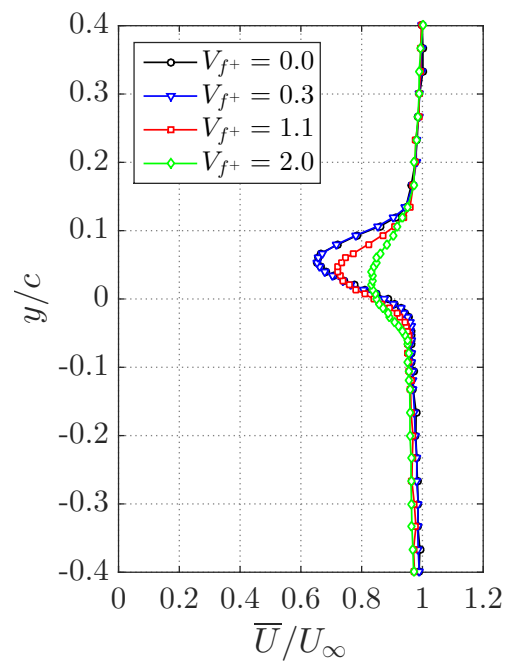

(a)

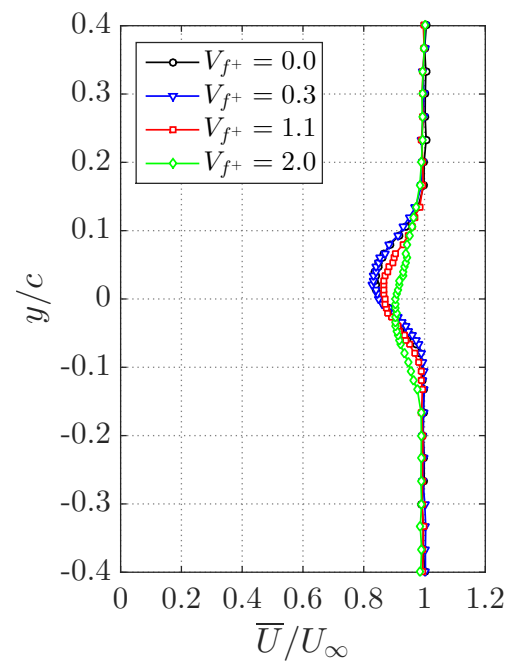

(b)

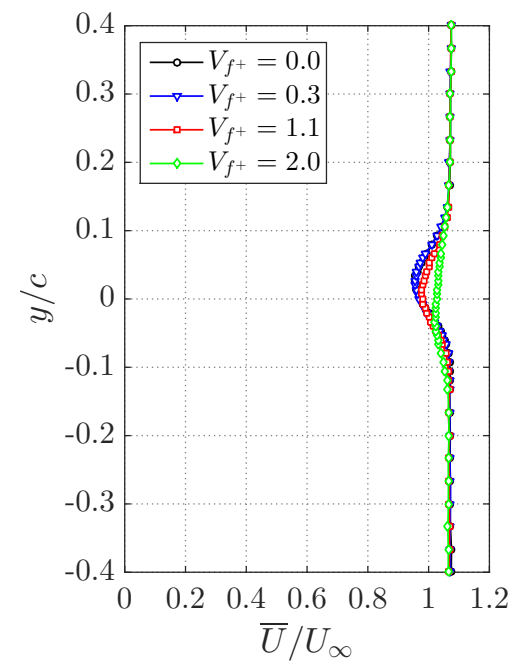

(c)

Figure 15: Profiles of $|U| / U_{\infty}$ in the wake: a) $1.25 c$, b) $1.50 c$, c) $1.70 c$.

\section{III.B.2. Aerodynamic Performance}

Figure 16 shows the force coefficents as a function of $\alpha$ and compares well with what has been described in section III.B.1. The $C_{L}$ curves belonging to the non-actuated and $V_{f^{+}}=0.3$ cases are very similar, as were the corresponding flow fields and wake profiles. At higher values of $V_{f^{+}}$the previously reported reduction in the size of the separated regions and velocity deficit have translated into significant improvements in performance. For example, when $V_{f^{+}}=2.0$ the regions of separation were reduced significantly at all values of $\alpha$ investigated and correspondingly a sizable increase in $C_{L}$ and decrease in $C_{D}$ is found at all $\alpha$ in figure 16 and this produces a large improvement in the $L / D$ ratio. Furthermore, figure 16b-c shows that when $V_{f^{+}}=1.1$ the effect on $C_{L}$ and $C_{D}$ increases with $\alpha$ which would support the greater reduction in separation at $\alpha=10^{\circ}$ and $15^{\circ}$ when compared with the lower angles. 


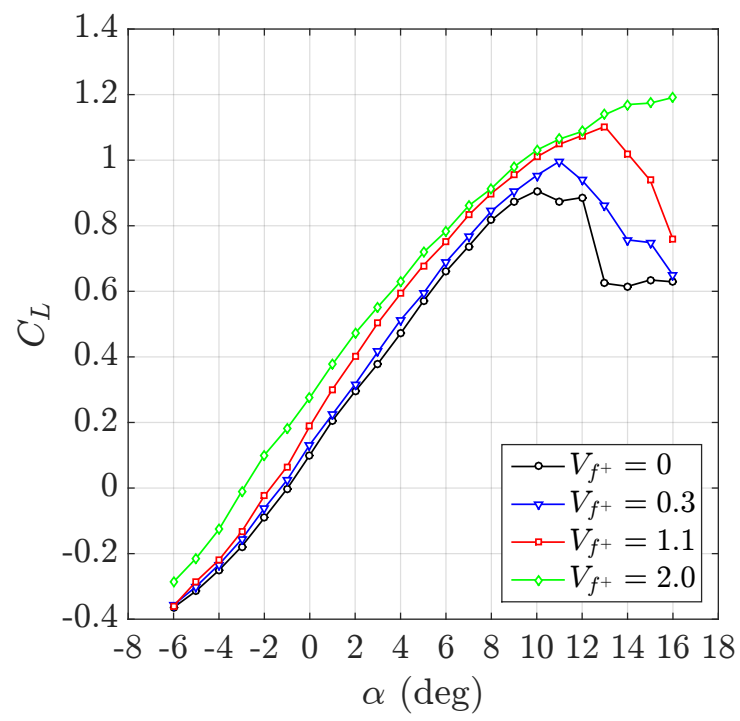

(a)

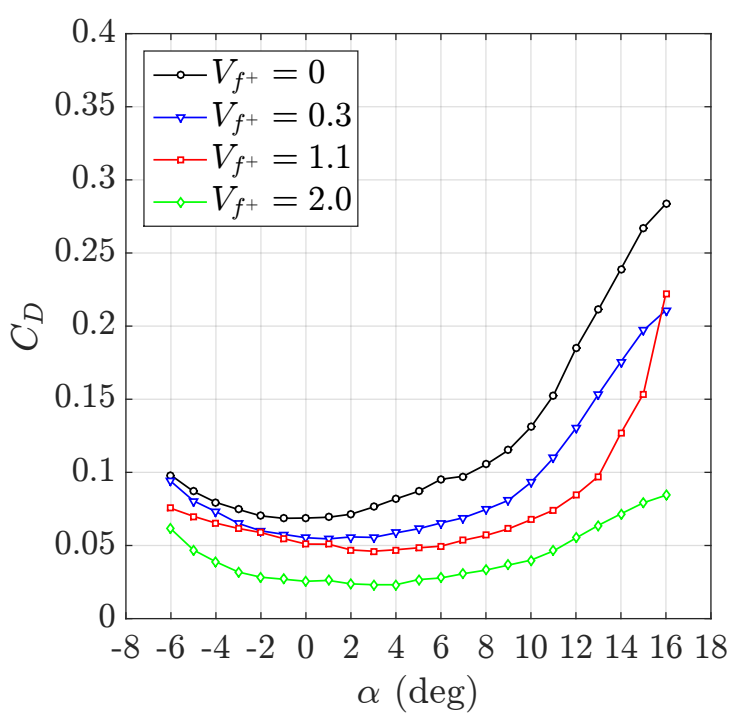

(b)

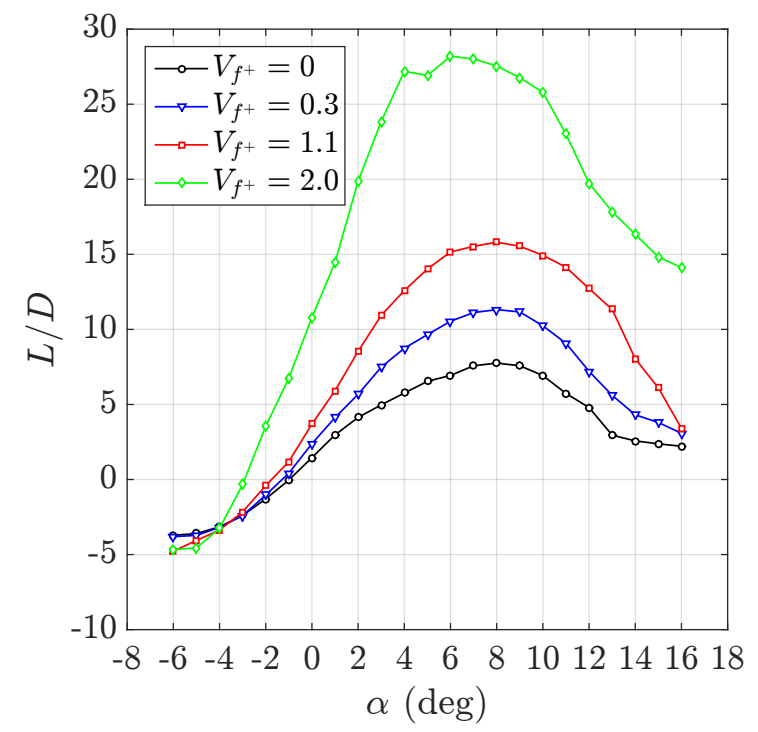

(c)

Figure 16: Force Coefficients as a function of $\alpha$ : a) $C_{L}$; b) $C_{D}$; c) $L / D$.

\section{III.B.3. Spectral Data}

Spectral data in the wake, seen in figure $17 \mathrm{~b}$ shows that the naturally occurring shedding frequency, when nondimensionalized by $U_{\infty}$ and $c$, is equal to a Strouhal Number, $S t_{c}$, of 2.4. When $V_{f^{+}}=0.3$ this shedding frequency is still present, as are the naturally occurring separation frequencies near the trailing edge seen in figure 17a. However, at frequencies approaching the shedding frequency, the actuation frequency begins to dominate the spectra in both the wake and separated region. When $V_{f^{+}}=1.1$, a peak at the separation frequency of roughly $S t_{c}=4.5$ is still visible in figure 17a despite the actuation frequency being strongly detected. However, when $V_{f^{+}}=2.0$ there are no natural frequencies still present. This suggests the control is most effective when it approaches the naturally occurring shedding frequency. The closer $V_{f+}$ gets the the shedding frequency, the more the flow locks-in to the surface motion and the more completely the natural development of the flow is destroyed. 


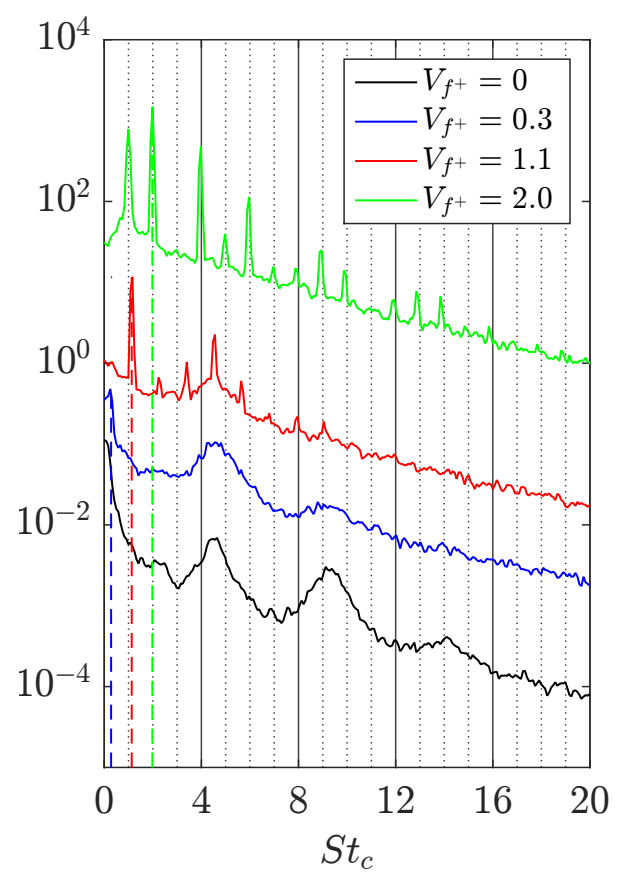

(a)

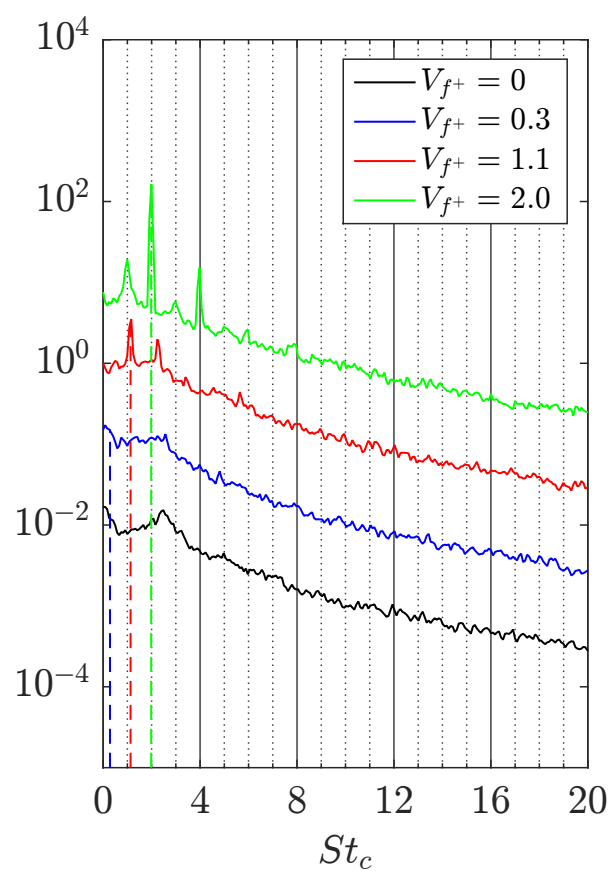

(b)

Figure 17: Frequency Spectra at the trailing edge of the airfoil and in the near wake when $\alpha=0^{\circ}$ : a) $0.9 c$; b) $1.25 c$. Dashed lines represent $V_{f^{+}}$.

The shedding frequency in the wake at different values of $\alpha$ is presented in figure 18. They show decrease a in shedding frequency as $\alpha$ increases and this could help explain the increased effect of low frequency actuation at higher angles of attack and is something that will be examined before the final manuscript is submitted.

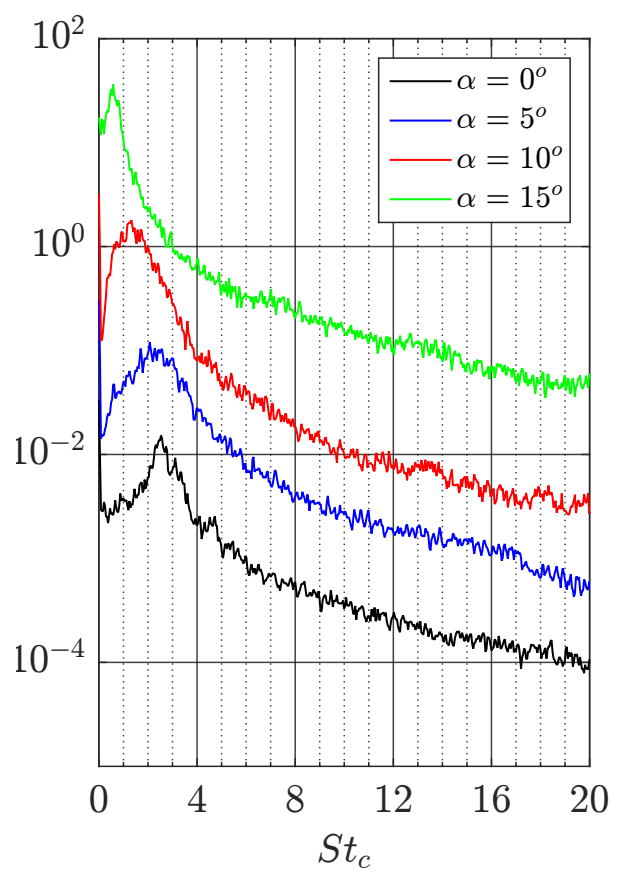

Figure 18: Frequency spectra at $1.50 \mathrm{c}$ with increasing $\alpha$ 


\section{Conclusion}

An airfoil model has been designed and constructed with an upper skin that can be dynamically actuated by applying a voltage signal to macro fiber composites (MFCs) bonded to its under side. These piezoelectric actuators are very thin, light and robust, and have low power consumption. The feasibility of applying these actuators for dynamic surface morphing was first investigated with a series of surface displacement measurements. It was found that the displacement depended on both frequency and voltage but the addition of flow was not found to change the skin's behavior. A variety of voltage amplitudes and frequencies were tested numerous times and the MFCs proved to be capable of accepting frequencies of at least $120 \mathrm{~Hz}$ at voltages of at least $400 \mathrm{~V}$. However, at all values of $V_{A}$ the peak-to-peak displacement was found to drop significantly at values of $V_{f}$ above $30 \mathrm{~Hz}$ and at $V_{f}$ greater than $100 \mathrm{~Hz}$ the nature of the displacement appears to change - possibly transferring into a different vibration mode - so for the purposes of this paper the frequency of actuation was kept below $90 \mathrm{~Hz}$. Extensive photogrammetry experiments were carried out to provide detailed 3D analysis of the surface motion at frequencies ranging from 10 to $100 \mathrm{~Hz}$. This data is being processed and it is intended that it will be included in the final manuscript.

The aerodynamic effect of the actuation was investigated with corresponding force balance, PIV and hotwire measurements. An effort was made to keep the amplitudes of displacement comparable by varying $V_{A}$ so the effect of frequency could be studied. It was found that at high values of $V_{f^{+}}$the flow locks-in to the surface motion which resulted in simultaneous increases in lift and decreases in drag. From the timeaveraged measurement around the airfoil and in the wake this performance improvement can be attributed to a reduction in the size of the separated region and a reduction in the velocity deficit.

Further analysis of the spectral data at different values of $\alpha$ will be performed for the final manuscript to further support the arguments regarding frequency lock-in and its relationship with the natural shedding frequency.

\section{Acknowledgements}

This research has been carried out at Imperial College London and Temasek Laboratories, National University of Singapore. The collaboration was made possible by an International Joint-PhD scholarship from Imperial College London's International Office and generous support from Temasek Laboratories. 


\section{References}

${ }^{1}$ Jones, G., Santer, M., Papadakis, G., Bouremel, Y., and Debiasi, M. T., "Open-Loop Flow Control At Low Reynolds Numbers Using Periodic Airfoil Morphing," 53rd AIAA Aerospace Sciences Meeting, American Institute of Aeronautics and Astronautics, Kissimmee, Florida, 012015.

${ }^{2}$ Mueller, T. J. and DeLaurier, J. D., "Aerodynamics of small vehicles," Annual Review of Fluid Mechanics, Vol. 35, No. 1, 01/01; 2013/04 2003, pp. 89-111.

${ }^{3}$ Postl, D., Balzer, W., and Fasel, H. F., "Control of laminar separation using pulsed vortex generator jets: direct numerical simulations," Journal of Fluid Mechanics, Vol. 676, 2011, pp. 81-109.

${ }^{4}$ Amitay, M., Smith, D., Kibens, V., Parekh, D., and Glezer, A., "Aerodynamic Flow Control over an Unconventional Airfoil Using Synthetic Jet Actuators," AIAA, Vol. 39, 2001, pp. 361-370.

${ }^{5}$ Seifert, A., Darabi, A., and Wygnanski, I., "On the Delay of Airfoil Stall by Periodic Excitation," Journal of Aircraft, Vol. 33, 1996, pp. 691699.

${ }^{6}$ Munday, D. and Jacob, J., "Active Control of Separation on a Wing With Oscillating Camber," Journal of Aircraft, Vol. 39, 1996, pp. 187-189.

${ }^{7}$ Debiasi, M., Bouremel, Y., Khoo, H. H., Luo, S. C., and Zhiwei, E. T., "Shape Change of the Upper Surface of an Airfoil by Macro Fiber Composite Actuators," 29th AIAA Applied Aerodynamics Conference, Honolulu, Hawaii, 062011.

${ }^{8}$ Bouremel, Y., L., C. W., Jones, G., and Debiasi, M. T., "Measurements of a Symmetric Airfoil Morphed by Macro Fiber Composite Actuators," 32nd AIAA Applied Aerodynamics Conference, American Institute of Aeronautics and Astronautics, Atlanta, Georgia, 062014.

${ }^{9}$ Debiasi, M., Bouremel, Y., Khoo, H. H., and Luo, S. C., "Deformation of the Upper Surface of an Airfoil by Macro Fiber Composite Actuators," 30th AIAA Applied Aerodynamics Conference, New Orleans, Louisiana, 062012.

${ }^{10}$ Boutilier, M. and Yarusevych, S., "Parametric study of separation and transition characteristics over an airfoil at low Reynolds numbers," Experiments in Fluids, Vol. 52, No. 6, 06 2012, pp. 1491-1506.

${ }^{11}$ Mueller, T. J. and Batil, S. M., "Experimental Studies of Separation on a Two-Dimensional Airfoil at Low Reynolds Numbers," AIAA Journal, Vol. 20, No. 4, 04 1982, pp. 457-463.

${ }^{12} \mathrm{O}$ 'Meara, M. and Mueller, T. J., "Laminar separation bubble characteristics on an airfoil at low Reynolds numbers," AIAA Journal, Vol. 25, No. 8, 08 1987, pp. 1033-1041. 